\title{
Atypical Polypoid Adenomyomas of Uterus: A Clinicopathologic Study of 2 Cases
}

\author{
Xiao Lin', Yu Li1 ${ }^{1,2,3^{*}}$ \\ ${ }^{1}$ Department of Pathology, Chongqing Medical University, Chongqing, China \\ ${ }^{2}$ Chongqing Key Laboratory of Neurobiology, Chongqing Medical University, Chongqing, China \\ ${ }^{3}$ Institute of Neuroscience, Chongqing Medical University, Chongqing, China \\ Email: *3100848087@qq.com
}

How to cite this paper: Lin, $\mathrm{X}$. and $\mathrm{Li}, \mathrm{Y}$. (2018) Atypical Polypoid Adenomyomas of Uterus: A Clinicopathologic Study of 2 Cases. Journal of Biosciences and Medicines, 6, 15-22.

https://doi.org/10.4236/jbm.2018.65003

Received: January 30, 2018

Accepted: May 18, 2018

Published: May 21, 2018

\begin{abstract}
Atypical polypoid Adenomyomas (APA) is a rare benign tumour in uterine. It is would distinguished from well-differentiated endometrial adenocarcinoma because of his histological features. We report 2 cases of 31-year-old and 33 -year-old patient whose symptom were abnormal uterine bleeding. The mass was pedunculated with a narrow pedicle connecting to the pedunculated with a narrow pedicle connecting to the cervical; the other was in the uterine prolapse without pedicle. The surgical procedure was polypectomy only in all two patients with hysteroscopy. The histopathology showed the lesions were composed of a proliferation of irregular atypical endometrial glands separated by varying amounts of cellular smooth-muscle stroma. No mitotic activity was observed in the epithelial or mesenchymal elements in APA. The treatment of APA should depend on the age and reproductive desire of patient.
\end{abstract}

\section{Keywords}

Atypical Polypoid Adenomyomas, Clinical, Pathology, Diagnosis

\section{Introduction}

Atypical Polypoid Adenomyomas (APA) is a kind of uterine benign lesion which was first designated by Mazur [1] in 1981. There were about one hundred cases have been reported in the world since 1981. APA is characterized by composing of irregular endometrial glands which show architectural complexity and cytological atypical of glandular epithelium, and they are mixed with a hyper cellular smooth muscle in the stroma, which is difficult to distinguish from well-differentiated endometrial carcinoma. Here we present the clinicopathological and immunohistochemical features of 2 cases of APA were diagnosed 
from 2010 to 2016 in Chongqing Medical University to discuss the clinicopathological and distinguished point of APA with combined of review of cases in the literature.

\section{Materials and Methods}

1) MATERIALS: 2 cases of APA were diagnosed between 2010 to 2016 at Department of Pathology in Chongqing Medical University.

2) METHODS: The formalin-fixed, paraffin-embedded tissues were sectioned at 5 um thickness for standard immunohistochemical staining. Slides were de-waxed for $15 \mathrm{~min}$ ( 2 times) in xylene, $5 \mathrm{~min}$ in absolute ethanol, $2 \mathrm{~min}$ in 95\% ethanol, 2 min in 80\% ethanol, and 5 min in distilled water, and then rehydrated into distilled $\mathrm{H}_{2} \mathrm{O}_{2}$ through graded ethanol. Antigen retrieval was used to enhance ER, PR, P53, KI67, CEA and h-caldesmon, SMA, Desmin, CD10 immunohistochemic by high press in citrate buffer ( $\mathrm{PH} \mathrm{6.0)}$ for $3 \mathrm{~min}$. Then the sections were washed and incubated those with over-night at $4^{\circ} \mathrm{C}$. Slides were washed in phosphate-buffered saline, and then incubated with secondary antibody for $20 \mathrm{~min}$ at room temperature. After washed, Slides were stained with $\mathrm{DAB}$ until desired stain intensity developed and mounted before observation by light microscopy.

\section{Results}

1) Clinical Findings: The age of patients in our report were 31 and 33 years old. The resenting symptoms were all abnormal uterine bleeding. Ultrasound examination showed a polyp at the lower part of the uterus in one case, prompting submucosal tumors. The other patient didn't take ultrasound examination before surgical procedure. One tumor was pedunculated with a narrow pedicle connecting it to the cervical; the other tumor was in the uterine prolapse without pedicle. The surgical procedure was polypectomy only in all two cases with hysteroscopy.

2) Pathologic Findings: The two lesions were all described as soft greytan and firm. The tumors were $1.5 \times 1.0 \times 1.0 \mathrm{~cm}, 1.0 \times 0.5 \times 0.5 \mathrm{~cm}$.

On low-power microscopic examination (Figure 1): The lesions were composed of a proliferation of atypical endometrial glands separated by varying amounts of cellular smooth-muscle Stroma. The boundary between the APA and adjacent stroma was usually sharp. Some irregular glands were lined with branched and back-to-back arrangements, and ranged from small simple glands to more irregularly shaped glands and large cysts. Cuff-like appearance was not found around irregular glands.

One high-power microscopic examination (Figure 1): Part of gland cells showed minimal to moderate nuclear atypical. Morularsquamous metaplasia was not present in all two cases. Small foci of intraluminal necrosis didn't occur in all two cases. Epithelial mitotic figures were rare in one case, but no atypical mitotic figure was identified. The stromal component consisted of smooth 


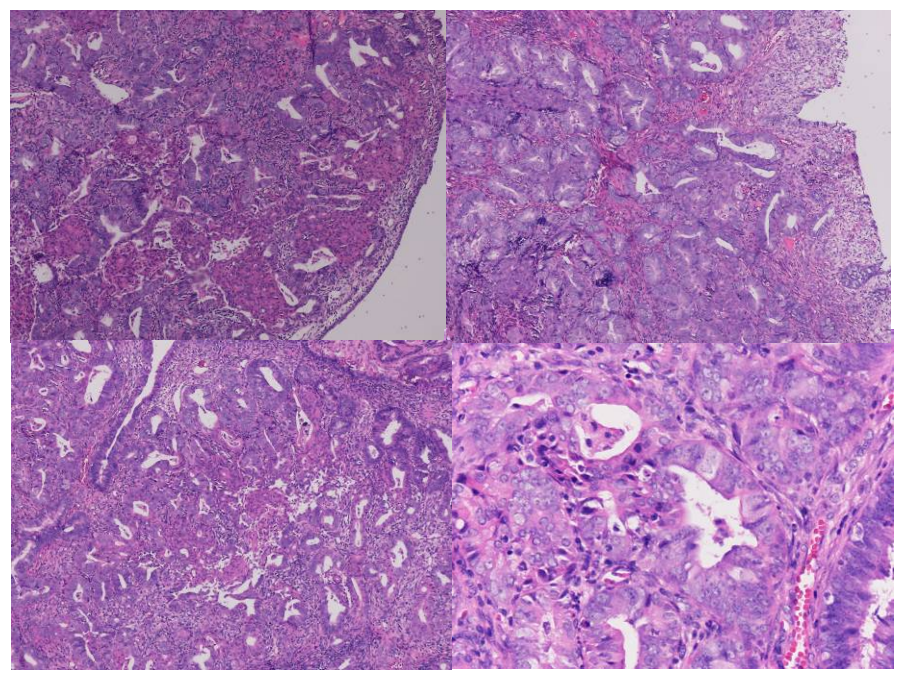

Figure 1. HE staining in APA.

muscle which was characterized by the typical small fusiform cells with eosinophilic cytoplasm and benign nuclear features . But the mitotic figures of cellular smooth-muscle were rare and did not exceed 2 per $10 \mathrm{hpt}$ when averaged over 40 hpt. Immunohistochemical staining (Figure 2): The stromal cells in all of the 2 APA Cases in which immunohistochemical staining was performed showed staining for SMA, Desmin but without CD10 and h-Caldesmon. The lesion glands showed prominent staining of the glandular epithelium with estrogen receptor(ER) and progesterone receptor(PR), but not showed P53 and CEA, the positive index of $\mathrm{Ki} 67<5 \%$.

\section{Discussion}

There were not more than 150 cases had been reported since 1981 when APA was named by Mazur. APA is a rare benign tumour in uterine. Recently, some scholar determined the APA to include high architectural index proliferations that lack extreme digress of nuclear polymorphism or nucleolus prominence and label them atypical polypoid adenomyofibroma of low malignant potential (APA-LMP). It was first named and defined as an uncommon benign polypoid endometrial tumor, which is composed of a proliferation of atypical endometrial glands with squamous metaplasia surrounded by cellular smooth muscle stroma. It is distinguished from well-differentiated endometrial adenocarcinoma because of his histological features. So, it is important purport for correct diagnosis and cure of APA to understand its features. We have expanded the clincopathologic definition of APA to include lesions with fibromatous stroma and architecturally complex glands in addition to those with a predominant smooth muscle stroma. The histogenesis of APA is poor understood. Some literates reported the causes of APA were suspected to be associated with obesity, hormone replacement therapy, prolonged estrogenic stimulation. APA may develop as a result of prolonged estrogenic stimulation was reported in some literature. [2] [3] [4] ongacre indicated that $9.1 \%$ of APA were treated with estrogenic or progestational 


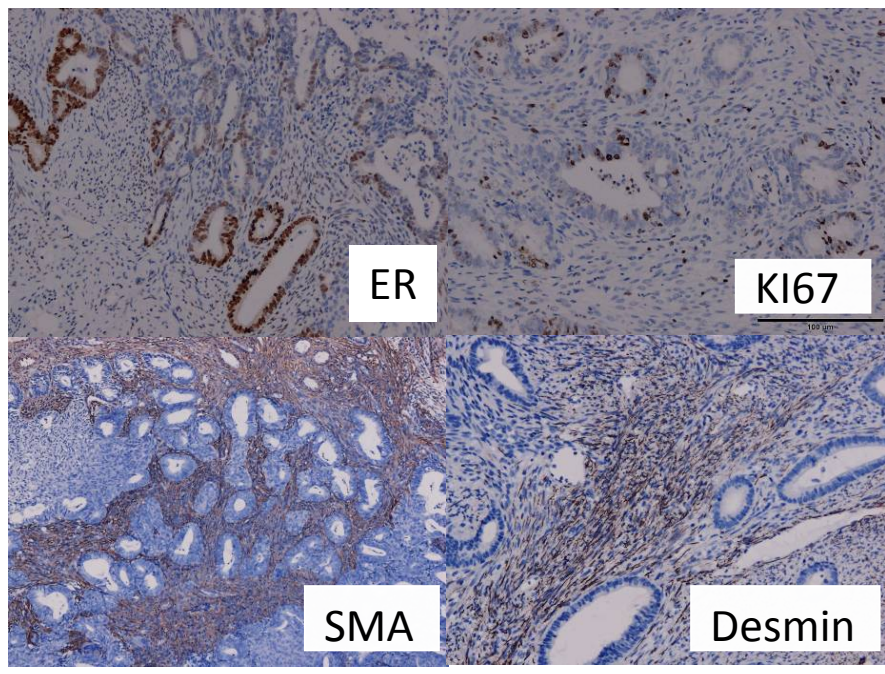

Figure 2. The positive staining of ER, KI67, SMA, desmin in APA.

therapy (oral contraceptive). 5.5\% of APA were stimulated ovulation/promoting ovulation/ovulation induction with tamoxifen therapy. 9.1\% of APA suffered from Turner syndrome. Zhou Qi [5] reported that endometrial glands cells were found to be express of ER and PR without treatment of hormone in 6 cases of APA which similar to 2 cases in our report. So, the relationship from APA with oral hormone is unclear.

The causes of APA were suspected to be associated with obesity, hormone replacement therapy, prolonged estrogenic stimulation, which were also the main suspected causes with endometrial carcinoma [6].

Clinical finding: The age of APA patients ranged from 21 to 73 years (mean 39.9 years); most of them ( $>90 \%)$ were at reproductive age and some were postmenopausal .The tumor measured from 0.1 to $6.0 \mathrm{~cm}$ in size at the lower segment of uterus. The patients with APA in our report were all childbearing age women.

The most common symptom was abnormal uterine bleeding, usually irregular menstrual bleeding, recurrent menorrhagia, or intermenstrual bleeding, some of patients have a clinical history of infertility. Less common symptoms included vaginal discharge, pelvic pain.

Ultrasound examination showed a polyp at the lower part of the uterus. Clinically, these lesions were most frequently interpreted as cervical polyps, fibroid, or polypoid carcinomas.

Pathologic feature: The lesions were usually described as gray, firm and bosselatea. The maximum dimensions of APAS varied from $0.1-9 \mathrm{~cm}$. On microscopic examination: The lesion was constituted of adenomyoma glands and smooth muscle. Architectural complexity adenomyoma glands distribution in apparent smooth muscle. The glands were variable in density and outline, some of these spaced with back-to-back arrangements, and ranged from small simple glands to more irregularly shaped glands and large cysts. They were lined predominantly by proliterative endometrioid-type epithelium. Glandular epithe- 
lium cells showed only minimal to moderate nuclear atypia in APA. Morular metaplasia was present in some of cases. The small foci of intraluminal necrosis were rare. The compartment was characterized by the typical small fusiform cells, with scant cytoplasm and benign nuclear features. No cuff-like express was seen. Occasional smooth muscle cells with bizarre nuclei were present. Rare cases contained scattered collections of chronic inflammatory cells consisting of small mature lymphocytes and rarely eosinophils or plasma cells. The distinguishing feature of APA as described by Mazur was a prominent cellular smooth-muscle stroma. However, Langacre has reported that fibrous tissue and cellular tissue are encountered in addition to the smooth muscle in some cases. Because the boundary separation the endometrial stroma phenotype and the uterine smooth-muscle phenotype has blurred in recent years. So, It had been adopted that the modified designation to emphasize the presence of a hybrid myofibromatous stroma in these proliferations. The stroma component consisted of smooth muscle and endometrial-type with smooth muscle predominating. The smooth muscle compartment was characterized by the typical small fusiform cells with scant cytoplasm and benign unclear features. Occasionally, smooth muscle cells with bizarre nuclei were present. No mitotic activity was observed in either the glandular, endometrial stromal, or smooth muscle. Abnormal mitotic figures were not observed.

Immunohistochemical staining: The adenomyoma gland cells in all of 2 APA cases in which immunohistochemical staining was preformed showed staining for ER and PR, ranging from weak to strong and diffuse, but KI67 < 5\%, P53(-). All of 2 cases showed prominent staining of the stroma cells with SMA and Desmin, without CD10 and h-caldesmon. It has been suggested that the immunoreactivity of APA stroma with SMA and desmin may help to distinguish these proliferations from the granulation tissue-like stroma of myoinvasive endometrial carcinoma [7]. Ohishi [8] et al. described the different immunohistochemical staining pattern of the CD10 in the stromal components of APA and myoinvasive endometrial carcinoma. In APA, CD10 was never or just focally and weakly positive expressed in the myofibromatous stroma, while all myoinvasive carcinomas expressed CD10 fringe-like staining pattern. Horita [9] found that CD10 was negative or weakly positive, whereas h-caldesmon was completely negative in the stromal cells of all 6 APAS. However, in the endometrioid carcinoma invaded myometrium, a fringe-like positive staining pattern was just occasionally observed for CD10, whereas a diffuse positive signal was obtained for h-caldesmon. They indicated that immunohistochemically, P53 and Ki67 are not reliable markers but that h-caldesmon is useful in distinguishing APA from myoinvasive endometriosis carcinoma.

Differential diagnosis: Compared with the myoinvasive well-Differentiated carcinomas, the APA samplings demonstrated a lower gland-to-stroma ratio and lower pronounced degree of glandular architectural complexity and cytological atypia. Key points of differential diagnosis: The age of well-differentiated endometrial adenocarcinoma is older than APA. Well-differentiated carcinoma 
whose tumors were used for comparison with the APA ranged in age from 48 74 years old (median 60). But about 55\% were aged about 40 years older or younger, $96 \%$ were premenopausal in APA. The lesions featured a biphasic proliferation of a architecturally complex and cytological irregular atypical endometrial glands within or desmin positive or both, but CD10 was negative. Ohishi described the CD10 was never or just weakly positive expressed in the myofibromatous stroma in APA, while was strong positive expressed in all myoimasive carcinoma [9]. Horita found that CD10 was negative in the stroma cells of APAS. However, in the endometrioid carcinoma invaded myometrium, staining pattern was occasionally observed for $\mathrm{CD} 10$, whereas a diffuse positive signal was obtained for h-caldesmon. They indicated that the P53, KI67 are not reliable markers but h-caldesmon was useful in distinguishing APA from myoinvasive endometrioid carcinoma.

The literatures reported that markedly complex, branching glands with morular metaplasia separated by thin intersecting fascicles of fibromusclular stroma. The Stroma in most cases consisted of broad bands of intersecting fibromuscular tissue, and the smooth muscle component of the stroma was predominant. On low-power micropic examination: The glands were variable in density and outline, but generally well spaced without density and outline, but generally well spaced without back-to-back arrangements, and ranged from small simple glands to more irregularly shaped glands and large cysts. They were lined by proliferative endometrioid-type epithelium. The differentiation of APA would be massive cyst, mature adipocytes and skeletal muscle differentiation [10] [11] [12]. The stromal component consisted of smooth muscle. The cellularity of the smooth muscle was variable. Marked hypercellularity of the smooth muscle, without cytological atypia was present in cases. No mitotic activity was observed in either the glandular, or smooth muscle component in most cases. The sarcomatous stroma is typically condensed around the neoplastic glands, at least focally resulting in a cuff-like appearance, an appearance that could be mimicked by the periglandular endometrial stroma in typical adenomyomas. Forever, when normal myometrium was invaded by carcinoma, there is more ordered and less cellular than the fibro muscular stroma of APA. An inflamed granulation tissue-like stroma is strongly associated with myoinvasive carcinoma in contrast to fibromuscular stroma, and showed raise the possibility of carcinoma. And there were foci of either marked architectural complexity or marked cytology atypia (or both) without an apparent smooth muscle or fibromuscular stromal in diagnosis of ECC. In short, the clinicopathologic features that include patient age, microscopic evidence of a focal process, the present of a biphasic glandular and fibromuscular stroma proliferation, architectural index.

Follow up: The two patients in our report were lost to follow-up. Longacre reported that the patients were all alive and well 1 to 112 months (mean, 25.2 months) after initial diagnosis in 54 of 55 patients with APA, and they found that 1 of the APAS with high architectural index and superficial myoinvasion was also associated with a well-differentiated endometrial carcinoma. And there 
were no extrauterine recurrences in the 55 APAS in Longacre's series. Fedele observed that the most patients treated with a conservative operation for adenomyomas were able to successfully conceive [13]. Literature reported that the incidence rate of endometrial carcinoma in APA was $8.8 \%$, but it was $24.3 \%$ $41.2 \%$ when the age of patients were older than 65 [14]. Though literatures reported that most patients coexisted with endometrial carcinoma (ECC) and the consecutive ECC was found just 1 year later after the initial APA diagnosis. Some believed that APA could represent an unusual form of precancerous endometrial proliferation similar to complex and atypical endometrial hyperplasia. However, we still could not draw a definite conclusion that APA all were premalignant lesions. We agree with the idea that APA were at least indicator lesions for malignancies.

Treatment: Maurizio [15] considered that the blunt curettage should not be performed either for the surveillance or for the treatment of APA, since it might miss or not completely remove the focal lesions and the differential diagnosis between APA and ECC with myometrial invasion may be difficult in a curettage specimen. Fedele et al. observed that most patients treated with a conservative treatment for adenomyomas were able to successfully conceive. So, the therapeutic principles of APA were: It was decided operation method according to the patients age and fertility requirements. Maurizio decided that four-step hysteroscopy technique for the treatment of APA was highly effective and convenient which included the removal of the APA, removal of endometrium adjacent to the APA, removal of the myometrium underlying the APA, and multiple random endometrial biopsies. It is important that be followed-up for patients with a conservative treatment.

In conclusion, APA should be carefully evaluated and cannot be automatically regarded as being a totally benign lesion. Additional cases are necessary reported for a better understanding of the natural history of APA and for providing appropriate management.

\section{References}

[1] Mazur, M.T. (1981) Atypical Polypoid Adenomyomas of the Endometrium. Am J Surg Pathol, 5, 473-482. https://doi.org/10.1097/00000478-198107000-00006

[2] Longacre, T.A., Chung, M.H., Rouse, R.V., et al. (1996) Atypical Polypoid Adenomyofibromas (Atypical Polypoid Adenomyomas) of the Uterus. A Clincopathologic Study of 55 Cases. Am J Surg Pathol, 20, 1-20. https://doi.org/10.1097/00000478-199601000-00001

[3] Bisceglia, M. (2002) Atypical Polypoid Adenomyoma. Adv Anat Pathol, 9, 256-260. https://doi.org/10.1097/00125480-200207000-00006

[4] Horn, L.C., Fischer, U. and Hockel, M. (2002) Polypoid Endometrial Adenomyoma in a Postmenopausal Woman, Mimicking Cervical Cancer, Associated with Ovarian the Coma. Areh Gynecol Obstet, 267, 101-103.

https://doi.org/10.1007/s00404-001-0253-8

[5] Zhou, Q., Ren, Y.Y. and Hou, W.L. (2013) Clinical Pathological Analysis of Endometrium Atypical Polyiform Adenomyoma. Medical Journal of Wuhan University, 
$34,552-554$.

[6] Fukuda, M., Sakura, I.N., Yamamoto, Y., Taoka, H., Asakawa, Y., Kurasaki, A., et al. (2011) Case of Atypical Polypoid Adenomyma That Possibly Underwent a Serial Progression from Endometrial Hyperplasia to Carcinoma. J Obstet Gynaecol Res, 37, 468-471. https://doi.org/10.1111/j.1447-0756.2010.01365.x

[7] Di Palma, S., Santini, D. and Martinelli, G. (1989) Atypical Polypoid Adenomyoma of the Uterus. An Immunohistochemical Study of a Case. Tumori, 75, 295. https://doi.org/10.1177/030089168907500322

[8] Ohishi, Y., Kaku, T., Kobayashi, H., et al. (2008) CD10 Immunostaining Distinguishes Atypical Polypoid Adenomyofibroma (Atypical Polypoid Adenomyoma) from Endometrial Carcinoma Invading the Myometrium. Hum Pathol, 39, 1446-1453. https://doi.org/10.1016/j.humpath.2008.02.006

[9] Horita, A., Kurata, A., Maeda, D., et al. (2011) Immunohistochemical Characteristics of Atypical Polypoid Sdenoma with Special Reference to h-Caldesmon. Int J Gynecol Pathol, 30, 64-70. https://doi.org/10.1097/PGP.0b013e3181efbe29

[10] Dobashi, Y., Fiedler, P.N. and Carcangiu, M.L. (1992) Polypoid Cystic Adenomyosis of the Uterus: Report of a Case. Int J Gynecol Pathol, 11, 240-243. https://doi.org/10.1097/00004347-199207000-00012

[11] Payne, F., Rollason, T.P. and Sirridis, E. (1992) Adenomyolipoma of the Endometrium-A Hamartoma? Histopathology, 20, 357-359. https://doi.org/10.1111/j.1365-2559.1992.tb00995.x

[12] Sink Te, P., Miller, D.S., Milchgrub, S. and Hameed, A. (2000) Adenomyofibroma of the Endometrium with Skeletal Muscle Differentiation. Int J Gynecol Pathol, 19, 280-283. https://doi.org/10.1097/00004347-200007000-00014

[13] Fedele, L., Bianchi, S., Zanotti, F., Marchini, M. and Candiani, G.B. (1993) Fertility after Conservative Surgery for Adenomyomas. Hum Reprod, 8, 1708-1710. https://doi.org/10.1093/oxfordjournals.humrep.a137919

[14] Heatley, M.K. (2006) Atypical Polypoid Sdenomyoma: A Systematic Review of the English Literature. Histopathology, 48, 609-610. https://doi.org/10.1111/j.1365-2559.2005.02315.x

[15] Maurizio, G., Greco, E., Di Spiezio Sardo, A., Di Carlo, C., Lavitola, G., Tarsitano, F., et al. (2008) Successful Pregnancy after Four-Step Hysteroscopic Technique for the Treatment of Atypical Polypoid Adenomyoma. Fertil Steril, 89, 1283-1284. https://doi.org/10.1016/j.fertnstert.2008.01.093 